\title{
1 Entropy Production by Earth System Processes
}

\author{
Axel Kleidon ${ }^{1}$ and Ralph Lorenz ${ }^{2}$ \\ 1 Department of Geography and Earth System Science Interdisciplinary Center, \\ 2181 Lefrak Hall, University of Maryland, College Park, MD 20742, USA \\ 2 Lunar and Planetary Lab, University of Arizona, Tucson, AZ 85721, USA
}

Summary. Degradation of energy to lower temperatures and the associated production of entropy is a general direction for Earth system processes, ranging from the planetary energy balance, to the global hydrological cycle and the cycling of carbon by Earth's biosphere. This chapter introduces the application of nonequilibrium thermodynamics to the planetary energy balance of Earth and its neighboring planets. The principles of minimum and maximum entropy production are introduced in the context of Earth system processes. Their applicability to the dynamics of the complex Earth system, such as atmospheric turbulence and the global biotic activity, is outlined. This chapter closes with an overview of the structure of the book and how the chapters relate to the overall theme of non-equilibrium thermodynamics.

\subsection{Introduction}

Earth system processes perform work by degrading sources of free energy, thereby producing entropy. For instance, the atmospheric circulation is slowed down by friction at the surface, so that it requires continuous input of work to maintain a steady-state circulation. The work to drive the atmospheric circulation is derived from the temperature gradient between the equator and the pole. The associated transport of heat from warmer to colder regions leads to a downgrading of the energy and entropy production. The global hydrological cycle is driven by energy conversions associated with evaporation from a warmer surface into an unsaturated atmosphere and subsequent condensation of water at a cooler temperature in the atmosphere. And life requires sources of free energy to build complex organisms. These three examples do not operate in isolation, but are highly interactive: Upward motion in the atmosphere often leads to condensation of water and cloud formation, so that the large-scale patterns of precipitation and uplift are highly correlated. Through its metabolisms, biotic activity substantially affects the chemical composition of the Earth's environment and physical characteristics of the land surface, such as surface albedo and aerodynamic roughness. All these examples involve transformations of energy of different forms, and these transformations are governed by the laws of thermodynamics. This leads us to the question whether thermodynamics can provide us with meaningful 
in: Kleidon, A. and Lorenz, R. D. (eds.) Non-equilibrium thermodynamics and the production of entropy: life, Earth, and beyond. Springer Verlag, in press.

(C) 2004 Springer Verlag, Heidelberg. ISBN: 3-540-22495-5

A. Kleidon and R. Lorenz

insights of how the steady-state of the complex Earth system operates at a macroscopic, planetary scale.

At the foundation of classical thermodynamics are the first and second laws. The first law formulates that the total energy of a system is conserved, while the second law states that the entropy of an isolated system can only increase. The second law implies that the free energy of an isolated system is successively degraded by diabatic processes over time, leading to entropy production. This eventually results in an equilibrium state of maximum entropy. In its statistical interpretation, the direction towards higher entropy can be interpreted as a transition to more probable states.

However, most systems are not isolated, but exchange energy and/or matter with their environment. For instance, planet Earth exchanges energy with its surroundings by radiation of different wavelengths. The formulations from classical thermodynamics can be applied to non-equilibrium systems which are not isolated (e.g., Prigogine 1962). By exchanging energy of different entropy (or mass) across the system boundary, these systems maintain states that do not represent thermodynamic equilibrium. For these systems, the second law then takes the form of a continuity equation, in which the overall change of entropy of the system $d S / d t$ is determined from the local increase in entropy within the system $d S_{I} / d t$ and the entropy flux convergence $d S_{E} / d t$ (i.e., the net flux of entropy across the system boundary):

$$
d S / d t=d S_{I} / d t+d S_{E} / d t
$$

In steady state, with no change of the internal entropy $S$ of the system, the production of entropy within the system $\sigma$ that leads to the increase $d S_{I} / d t$ balances the net flux of entropy across the system boundary $d S_{E} / d t$. The second law in this form then states that $\sigma \geq 0$. A non-equilibrium system can maintain a state of low entropy by "discarding" high entropy fluxes out of the system.

\subsection{Entropy Production of Climate Systems}

The Earth is a non-equilibrium system in a steady state. At the planetary scale, the absorption of solar radiation is balanced by the emission of terrestrial radiation, leading to the following planetary energy balance:

$$
I_{0}\left(1-\alpha_{\mathrm{P}}\right)-\sigma_{\mathrm{B}} T_{R}^{4}=0
$$

with $I_{0}$ being the net flux of solar radiation, $\alpha_{\mathrm{P}}$ being the planetary albedo, $\sigma_{\mathrm{B}}$ being the Stefan-Boltzmann constant, and $T_{R}$ the effective radiative temperature. Using present-day values for Earth of $I_{0}=342 \mathrm{~W} \mathrm{~m}^{-2}$ and $\alpha_{\mathrm{P}}=0.3$ one obtains a net radiative temperature of $T_{R}=255 \mathrm{~K}$. 


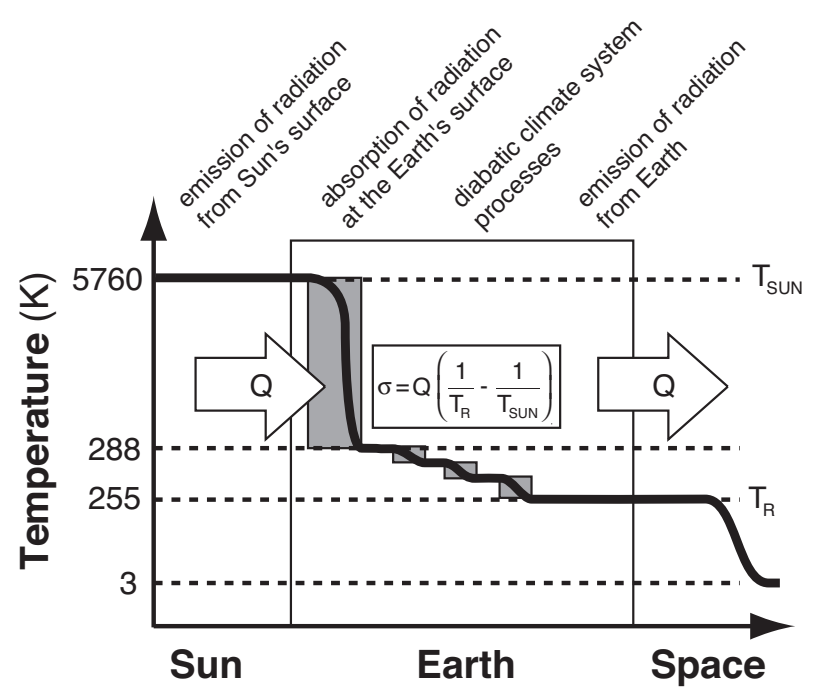

Fig. 1.1. Schematic diagram showing how free energy is subsequently degraded by processes within the Earth system to subsequently lower temperatures

\subsubsection{Earth's Climate System}

As the flux of energy passes through the Earth system, it is subsequently degraded to lower temperatures, leading to the production of entropy (Fig. 1.1). Solar radiation, emitted at a high radiative temperature of the Sun (of about $\left.T_{S U N}=5760 \mathrm{~K}\right)$, represents a flux of low entropy. It consists of a flux of photons of high energy, that is, the emitted energy is concentrated on relatively few photons, each carrying a large amount of energy. When solar radiation is absorbed at the Earth's surface at a surface temperature of roughly $T_{S} \approx 288 \mathrm{~K}$, entropy is being produced in the amount of:

$$
\sigma_{\mathrm{RAD}}=Q\left(1 / T_{S}-1 / T_{S U N}\right)
$$

with $Q$ being the amount of radiation being absorbed. Further transformations of the energy take place at subsequently lower temperatures. For instance, the latent heat flux removes energy from the surface at a temperature $T_{S}$ and is released to the atmosphere at a lower temperature $T_{A}$, leading to entropy production in the amount of:

$$
\sigma_{\mathrm{LH}}=Q_{L H}\left(1 / T_{A}-1 / T_{S}\right)
$$

Similarly, the sensible heat flux, the absorption of terrestrial radiation in the atmosphere, and the transport of heat from warmer to colder regions by the atmosphere and the oceans also contribute to the production of entropy. Ultimately, the absorbed solar radiation is reemitted into space as terrestrial 
radiation at roughly the radiative temperature $T_{R}$. In contrast to solar radiation, this radiation is emitted from Earth at a much lower temperature, representing a flux of photons of less energy and high entropy. The overall entropy production of the Earth system can be estimated from the difference of entropy fluxes across the Earth-space boundary:

$$
\sigma_{\mathrm{TOT}}=I_{0}\left(1-\alpha_{\mathrm{P}}\right)\left(1 / T_{R}-1 / T_{S U N}\right) \approx 900 \mathrm{~mW} \mathrm{~m}^{-2} \mathrm{~K}^{-1}
$$

By using the energy fluxes of the global energy balance and estimates for the respective temperatures at which the transformations of energy occur, one can derive the contribution of various diabatic processes of the climate system to the overall production of entropy. Figure 1.2 shows a global estimate of entropy production by different, climate-related processes, based on the analysis of Peixoto et al. (1991) (see also Goody, 2000). From Fig. 1.2 it is evident that the greatest amount of entropy is generated by the absorption of solar radiation in the atmosphere and at the surface. An order of magnitude less is the entropy production associated with the absorption of longwave radiation in the atmosphere and by the transfer of latent heat from the surface to the atmosphere associated with the global hydrological cycle. Seemingly small are the contributions that originate from the sensible heat flux and the frictional dissipation associated with the atmospheric circulation. What Fig. 1.2 shows us is that the overall production of entropy by the Earth system is most profoundly affected by changes in absorption of solar radiation through the planetary albedo, and to a lesser extent by the partitioning of energy at the surface into radiative and turbulent fluxes, and the dynamics of the atmospheric circulation. Since the radiative temperature of the Earth, $T_{R}$, is primarily determined by the planetary energy balance, $T_{R}$ plays a relatively minor role.

\subsubsection{Other Planetary Climate Systems}

Planets other than the Earth can be considered in an analogous way, as pointed out by Aoki (1983). Catling (this volume) makes the observation that of the planets with atmospheres in our solar system, it is the Earth that has the highest entropy generation rate, perhaps not coincidental with its being an abode for enduring life (Table 1.1). The radiative settings (insolation, albedo and atmospheric opacity) are different for different planetary bodies, yet as discussed later (Sect. 1.3.3 and in Lorenz, this volume) the Maximum Entropy Production principle that appears to drive equator-topole heat transport on Earth appears to apply at least to Mars and Titan. Much work remains to quantify the entropy budgets that may pertain on other planets - for example, the heat transports on Mars and Neptune's moon Triton are dominated by the latent heat associated with seasonal sublimation and interhemispheric migration of carbon dioxide and nitrogen respectively. 
in: Kleidon, A. and Lorenz, R. D. (eds.) Non-equilibrium thermodynamics and the production of entropy: life, Earth, and beyond. Springer Verlag, in press.

(c) 2004 Springer Verlag, Heidelberg. ISBN: 3-540-22495-5

1 Entropy Production by Earth System Processes

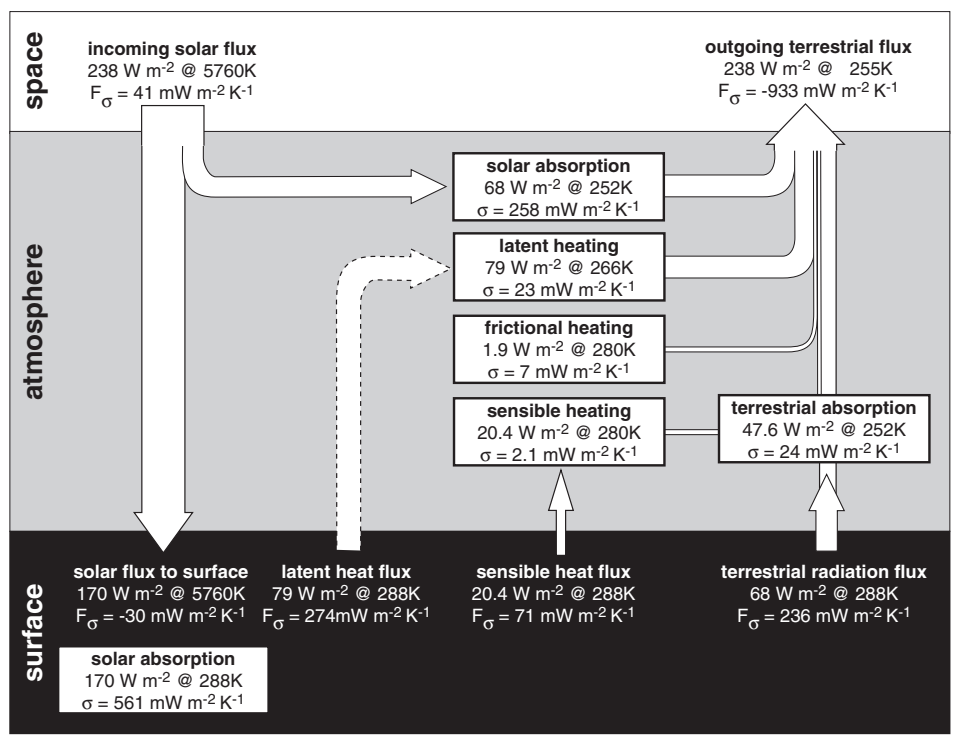

Fig. 1.2. Global budget of entropy production by diabatic Earth system processes. Based on Peixoto et al. (1991). Note that the latent heat flux has been corrected from Peixoto et al.'s estimate

Table 1.1. Planetary entropy production of Earth in comparison to Venus and Mars. Based on data from the National Solar System Data Center (http://nssdc.gsfc.nasa.gov/planetary)

\begin{tabular}{llll}
\hline & Venus & Earth & Mars \\
\hline Luminosity $\left(\mathrm{W} \mathrm{m}^{-2}\right)$ & 2614 & 1368 & 589 \\
Planetary Albedo & 0.75 & 0.31 & 0.25 \\
Black-body Temperature $(\mathrm{K})$ & 232 & 254 & 210 \\
Entropy Production $\left(\mathrm{mW} \mathrm{m}^{-2} \mathrm{~K}^{-1}\right)$ & 676 & 893 & 507 \\
\hline
\end{tabular}

\subsection{The Principles of Minimum and Maximum Entropy Production}

Diabatic processes do not produce entropy at an arbitrary rate. Two relevant extremum principles have been formulated to describe the characteristic behavior of non-equilibrium systems. For systems near thermodynamic equilibrium with fixed boundary conditions, Prigogine (1962) formulated the principle of minimum entropy production (MinEP) stating that the steady state of the process is associated with a MinEP state. However, many processes do not have fixed boundary conditions and are far from equilibrium. For those processes it has been shown from information theory (Dewar 2003; also Dewar, this volume) that these processes maintain steady states in which the production of entropy is maximized (MaxEP, or MEP) if there are suffi- 
in: Kleidon, A. and Lorenz, R. D. (eds.) Non-equilibrium thermodynamics and the production of entropy: life, Earth, and beyond. Springer Verlag, in press.

(C) 2004 Springer Verlag, Heidelberg. ISBN: 3-540-22495-5

A. Kleidon and R. Lorenz

cient degrees of freedom associated with the process (what the specific degrees of freedom are depends on the particular circumstances of the process, see below). Instead of providing formal derivations of the two principles, heat transport is used as an example to highlight the applicability of these two very different principles in the following.

Note that some confusion can arise in how the phrase 'Maximum Entropy Production' is parsed. The attainment of a state of Maximum Entropy (i.e., equilibrium) is a well-accepted one, and Maximum-Entropy methods are widely accepted as estimation techniques. The tendency of systems which are in a steady state, but one that is held away from equilibrium by an external input of energy, to produce entropy at a maximum possible rate, is what we generally mean by 'Maximum Entropy Production'. Clearly there are interesting relationships between the two ideas, as discussed in this book, but it is important to bear the distinction in mind (see also discussion on Shannon information entropy and Maximum Entropy Production in Dewar, this volume).

\subsubsection{Heat Transport and Minimum Entropy Production}

A simple formulation of heat transport from a warm reservoir with a fixed temperature $T_{W}$ to a cold reservoir with a fixed temperature $T_{C}$ can be used to illustrate the MinEP principle. The change of temperature $T_{M}$ at a location between the two reservoirs is described by the difference of heat fluxes at this location:

$$
d T_{M} / d t=Q_{W}-Q_{C}
$$

with the heat fluxes from the warm reservoir $Q_{W}$ and to the cold reservoir $Q_{C}$ expressed as:

$$
\begin{gathered}
Q_{W}=k\left(T_{W}-T_{M}\right) \\
Q_{C}=k\left(T_{M}-T_{C}\right)
\end{gathered}
$$

The rate of entropy production associated with heat transport $\sigma_{H T}$ is written as:

$$
\sigma_{H T}=Q_{W}\left(1 / T_{M}-1 / T_{W}\right)+Q_{C}\left(1 / T_{C}-1 / T_{M}\right)
$$

Figure 1.3 shows the rate of entropy production as a function of $T_{M}$. The steady-state is achieved with $Q_{W}=Q_{C}$ which leads to zero change of $T_{M}$ with time (1.6). The steady state is associated with minimum amount of entropy production as shown in Fig. 1.3 (for small $T_{W}-T_{C}$ ). This trivial result can be obtained from the entropy minimization procedure, but since the boundary temperatures are fixed, the result follows from the assumption of steady-state in any case. 


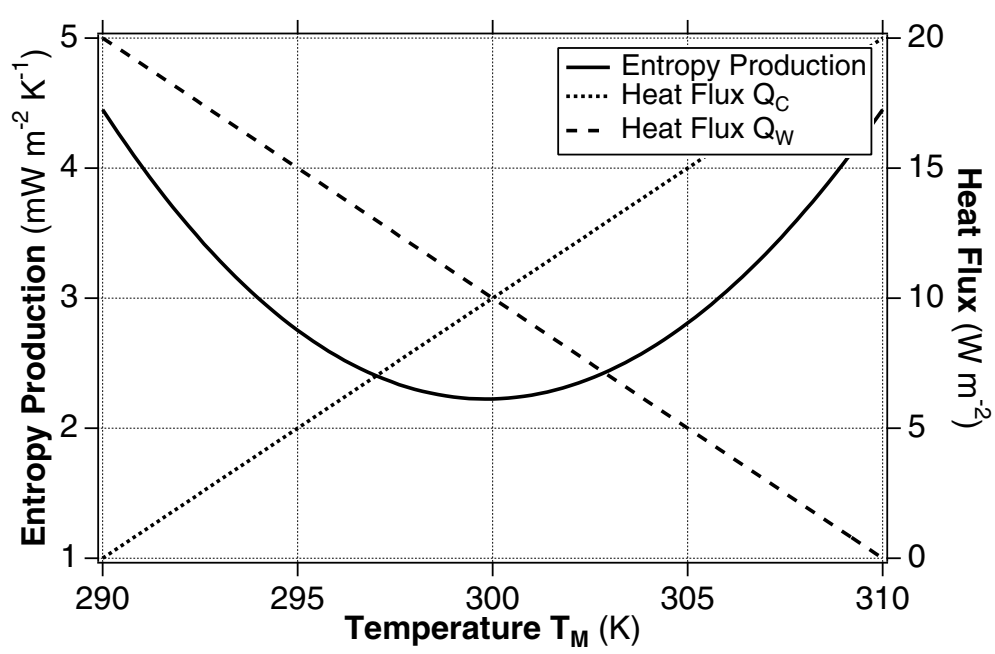

Fig. 1.3. Minimum Entropy Production and heat transport with fixed boundary conditions. Arbitrary conditions of $T_{W}=310 \mathrm{~K}, T_{C}=290 \mathrm{~K}$, and $k=1 \mathrm{~W} \mathrm{~m}^{2} \mathrm{~K}^{-1}$ are used

Miyamoto et al. (this volume) describe how a dissipation minimization procedure can be used to form efficient river networks in model hydrological systems (also, Rinaldo et al. 1996). In these systems, the boundary conditions are fixed (equal amount of rain in each cell of a grid: all the flow exits the network through one cell at the corner of the grid so the system is a network which connects all cells to that corner). These fixed boundary conditions make a minimization rather than a maximization the appropriate procedure to apply, and yield networks comparable with (but not quite the same as) river networks observed in nature. We may note that these models are rather analogous to the studies of Bejan (2000), who shows how entropy production minimization can be applied to optimize the design of heat transfer systems (where heat is produced uniformly in a volume or area and must be conveyed to a heat sink at one corner or edge). Although not invoking MinEP, similar scaling relationships to those found for river networks have been derived from fractal geometry for distribution networks of individual, living organisms and plant communities (e.g., Enquist et al. 1998; West et al. 1999).

\subsubsection{Heat Transport and Maximum Entropy Production}

When we want to describe the heat transport from the tropics to polar regions on Earth, we cannot assume fixed boundary conditions as required by the MinEP principle. The temperatures of the tropics and the poles are determined from the local energy balances, which in turn are affected by the amount of heat that is transported from the tropics to the poles. Also note that $k$ is not a fixed physical property that can be easily measured, since 
in: Kleidon, A. and Lorenz, R. D. (eds.) Non-equilibrium thermodynamics and the production of entropy: life, Earth, and beyond. Springer Verlag, in press.

(C) 2004 Springer Verlag, Heidelberg. ISBN: 3-540-22495-5

A. Kleidon and R. Lorenz

heat transport in the climate system is primarily associated with the turbulent motion of large-scale eddies in the atmosphere and oceans. Therefore, $k$ is determined from the characteristic properties of fluid turbulence in a macroscopic steady state. Under these conditions, it has been proposed that heat transport adjusts itself such that the production of entropy in steady state is at a maximum. The existence of a maximum in entropy production associated with poleward heat transport can easily be demonstrated with a simple energy balance model of the climate system, following earlier work by Lorenz (1960), Paltridge (1975) and others.

We use two boxes to represent the local energy balances of tropical and polar regions and allow for heat transport between the two boxes. The only processes considered are the absorption of solar radiation in the tropics $Q_{I N, T}$ and in the polar regions $Q_{I N, P}$, the emission of terrestrial radiation $Q_{O U T}$, taken as $a+b T$, and the transport of heat between the boxes $Q_{H T}$. With these simplifications, we can write the energy balances for the two boxes as

$$
\begin{aligned}
& I_{T}-\left(a+b T_{T}\right)-Q_{H T}=0 \\
& I_{P}-\left(a+b T_{P}\right)+Q_{H \mathrm{~T}}=0
\end{aligned}
$$

with the empirical coefficients $a=204 \mathrm{~W} \mathrm{~m}^{-2}$ and $b=2.17 \mathrm{~W} \mathrm{~m}^{-2} \mathrm{~K}^{-1}$ (Budyko 1969; Sellers 1969).

We can express the heat transport term $Q_{H T}$ as a diffusive flux as:

$$
Q_{H T}=k\left(T_{T}-T_{P}\right)
$$

with $k$ being an effective heat conductivity. The transport of heat from a warmer, tropical reservoir to colder polar regions leads to entropy production, $\sigma_{\mathrm{HT}}$ given by:

$$
\sigma_{\mathrm{HT}}=Q_{H T}\left(1 / T_{P}-1 / T_{T}\right)
$$

In traditional applications of energy balance models (e.g., Budyko 1969; Sellers 1969), the value of $k$ is kept at a fixed value. The MEP hypothesis suggests that the turbulent motion of the atmosphere and oceans adjusts in such a way that $\sigma_{\mathrm{HT}}$ is maximized with respect to $k$, subject to external constraints such as the conservation of energy, mass and momentum.

Figure 1.4 shows $\sigma_{\mathrm{HT}}$ as a function of $k$, with a maximum value of $\sigma_{\mathrm{HT}}$ for $k \approx 2 \mathrm{~W} \mathrm{~m}^{-2} \mathrm{~K}^{-1}$. This value is close in magnitude to the commonly used value in energy balance models of North and others (e.g., North et al. 1981). The maximum in entropy production is the result of the competing effects of enhanced heat transport $Q_{H T}$ and reduced temperature gradient $T_{T}-T_{P}$ on $\sigma_{\mathrm{HT}}$ with increasing values of $k$ (Fig. 1.4). The planetary rate of entropy production $\sigma_{T O T}$ also increases with increasing $k$, since enhanced heat transport leads to a more uniform distribution of temperature, resulting in a reduction of the overall net radiative temperature $T_{R}$. 
in: Kleidon, A. and Lorenz, R. D. (eds.) Non-equilibrium thermodynamics and the production of entropy: life, Earth, and beyond. Springer Verlag, in press.

(C) 2004 Springer Verlag, Heidelberg. ISBN: 3-540-22495-5

1 Entropy Production by Earth System Processes

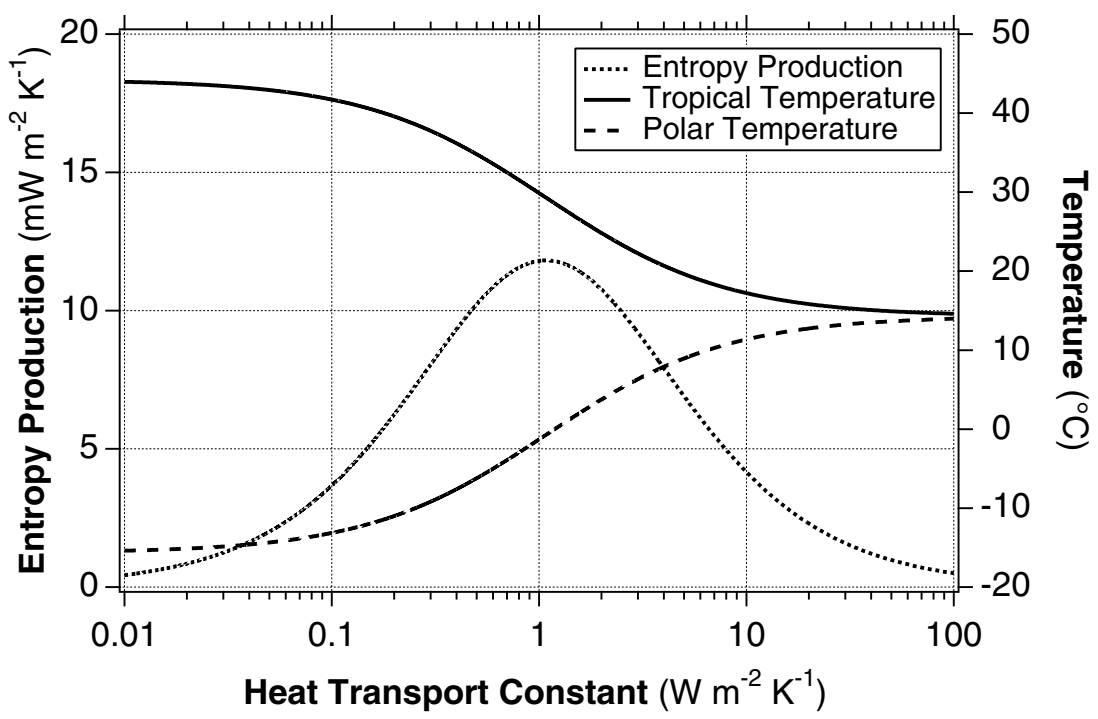

Fig. 1.4. Equator-Pole temperature gradient, and entropy production as a function of heat transport coefficient

At the state of MEP, the atmospheric circulation responds primarily with negative feedbacks to external perturbations. This can be illustrated by considering how the driver of heat transport, that is, the temperature difference between the equator and the pole, reacts to external perturbations. Imagine a fluctuation that leads to a temporary increase in the heat transport coefficient. This leads to a increased warming of the pole at the expense of a stronger cooling in the tropics. Therefore, the equator-pole temperature gradient is reduced, leading to a lower thermodynamic efficiency that uses that heat transport to drive the circulation, resulting in a negative feedback to the perturbation. A similar case can be made for a fluctuation that leads to a temporary decrease in heat transport.

What Lorenz (1960), Paltridge (1975, 1978, 2001) and others (see e.g., review by Ozawa et al. 2003) showed with more detailed energy balance model simulations is that several observed features of the climate system, such as the intensity of the atmospheric circulation, the equator-pole temperature difference in surface temperature, and the meridional distribution of cloud cover reflect a state of the climate system which is close to a state of MEP.

Recently, the MEP principle has been confirmed by simulations with general circulation models that explicitly simulate the fluid dynamics that lead to turbulence (Shimokawa and Ozawa 2001, 2002; Kleidon et al. 2003). Shimokawa and Ozawa (2001, 2002, also this volume) showed with an ocean general circulation model that of the stable steady states of the model, the system assumes the one with the highest rate of entropy production after perturbation. Kleidon et al. (2003) showed with atmospheric general circulation 
in: Kleidon, A. and Lorenz, R. D. (eds.) Non-equilibrium thermodynamics and the

production of entropy: life, Earth, and beyond. Springer Verlag, in press.

(C) 2004 Springer Verlag, Heidelberg. ISBN: 3-540-22495-5

A. Kleidon and R. Lorenz

model simulations that increasing the model's spatial resolution increases entropy production up to a certain value after which entropy production is not further increased (also Ito and Kleidon, this volume). They interpreted these results along the lines of Dewar's (2003) interpretation of MEP: spatial resolution affects the spatial degrees of freedom of the atmospheric flow, and therefore entropy production should increase up to the point at which sufficient degrees of freedom are represented in the model.

The confirmation of the MEP principle can be of great utility to Earth system science. Shimokawa and Ozawa's (2001, 2002) results demonstrate that one can assign probability to multiple steady states, and that the MEP state is the most likely state of the climate system. In this context, MEP may help us to constrain possible climatic states of the Earth's past, for which broad-scale observations often lack. Kleidon et al.'s (2003) study motivate the use of MEP as a consistency check for numerical models, in terms of defining a minimum spatial resolution that should be used to adequately simulate large-scale turbulence in the mid-latitude atmosphere, or for tuning simple model parameterizations, such as boundary layer turbulence, for which the degrees of freedom are not explicitly simulated in the model. In fact, if MEP states are not represented by model simulations of the climate systems, that is, that the simulated climate system does not work as hard as it could, it is likely to lead to model biases and misrepresentations of the climate sensitivity to global change (Grassl 1981; Kleidon et al. 2003).

\subsubsection{Maximum Entropy Production in a Planetary Context}

MEP is of great utility where there is little information to characterize the system's state, particularly of other planets. Lorenz et al. (2001) studied the zonal climates of the planet Mars, and of Saturn's moon Titan with a simple two-box model like that above, and found that the equator-to-pole temperature difference observed is consistent with the MEP hypothesis. Observed temperatures require, and MEP predicts a value of $k$ (often referred to in zonal EBMs as ' $D$ ') for Mars that is rather similar to Earth's, and a much smaller value for Titan. These results are rather surprising, in that Mars' atmosphere is very thin, and thus would be expected from dynamical arguments to transport less heat, while Titan's atmosphere is thicker than Earth's and so should transport more heat (especially considering that body's small physical size and the fact that it rotates slowly, a dynamical regime that should encourage efficient equator-to-pole circulations.) MEP does not provide, however, an explanation for these results - it only suggests what the net effect of all transport processes should be. In the case of Mars, it seems that the heat transport can be so large in the thin atmosphere because the latent heat of the Martian $\mathrm{CO}_{2}$ frost cycle can carry the bulk of the heat required by MEP.

As discussed by Lineweaver (this volume) and Chaisson (this volume), the temperature of the Universe as a whole - the heat sink to which the planets 
reject heat - has evolved through time. Although the universe was initially prohibitively hot to permit the efficient rejection of heat from evolving systems, the temperature fell to a few tens of $\mathrm{K}$ - more or less comparable with the present $3 \mathrm{~K}$ cosmic background - at the same time that matter began to accumulate into planets. The further evolution of the Universe is based on this temperature differential and allows for the evolution towards higher rates of entropy production and the creation of more complex structures. Certain aspects of this evolution should clearly be reproducible at a macroscopic scale in a thermodynamic sense.

In addition to the bulk transport state of the fluid system, the informationtheoretic or probabilistic entropy of the detailed configuration of the system can be a useful exploratory and predictive tool. Sommeria (this volume) shows how applying an entropy maximization procedure to the vorticity distribution of a flowfield such as the circulation of Jupiter's atmosphere can yield remarkable results, such as the emergence of large long-lived vortices like the Great Red Spot.

Thus MEP has a predictive utility for poorly-known environments - only the radiative setting (i.e., insolation and the greenhouse effect) need be known. It may be that the principle is useful for planetary interiors and perhaps for dynamic systems such as planetary rings.

\subsubsection{Minimization Versus Maximization of Entropy Production}

In summary, it is important to emphasize the differences between the MinEP and the MEP principles. The MinEP principle, as discussed above, applies to linear systems with fixed boundary conditions and few degrees of freedom, and concerns situations where such a system is perturbed away from its steady state. The steady state is one of minimum entropy production relative to any adjacent non-steady state. In contrast, the MEP principle applies to nonlinear systems with many degrees of freedom which allow the existence in principle of multiple steady states. The 'chosen' steady state has maximum entropy production relative to the other possible steady states. It is important when dealing with MEP that constraints such as the conservation of energy, mass and momentum are adequately considered in any specific application.

The MEP principle is not without controversy. There have been three prominent objections to MEP raised by meteorologists. The first objection is that there was no apparent justification for the system 'wishing' to find the MEP state. This objection appears to be addressed by the work of Dewar (2003), and the extension of maximum entropy ideas more generally, and by the notion of negative feedbacks associated with MEP states (e.g., Ozawa et al. 2003).

A second objection is that MEP cannot always apply, in that some atmospheres will be too thin to transport the required amount of heat without violating some physical constraint such as the speed of sound (e.g., Rodgers 1976). Rodgers suggested that this would apply to Mercury and Mars. In 
in: Kleidon, A. and Lorenz, R. D. (eds.) Non-equilibrium thermodynamics and the production of entropy: life, Earth, and beyond. Springer Verlag, in press.

(C) 2004 Springer Verlag, Heidelberg. ISBN: 3-540-22495-5

A. Kleidon and R. Lorenz

the latter case, the latent heat of the frost cycle appears to make up for the atmosphere's thinness, and so the objection does not hold. For Mercury, the argument does indeed apply. This objection relates to the conditions laid out above, that MEP is subject to the system constraints.

A similar and more widely-expressed argument is that the heat transport mandated by MEP is independent of the planetary rotation rate. As discussed by Ito and Kleidon (this volume), the constraints associated with the Earth's rotation rate are explicitly considered in climate models and should help to quantify the applicability of MEP. On a rapidly-rotating planet, the circulation required to transport the heat would be dynamically unstable, or would require too much mechanical work generation to sustain against frictional dissipation. Thus, a heat transport lower than that sought by MEP would be observed. However, for very slowly-rotating planets (i.e., where the flow is not constrained by rotational dynamics) MEP should apply, and in any case will specify the maximum possible transport given the constraints of the system.

\subsection{Entropy Production and Life on Earth}

Every living organism depends on a source of free energy that can be utilized by its metabolism and used to do work (grow, move, reproduce). The basis for every metabolic pathway is a source of free energy provided by the environment. In the words of Schrödinger (1944), life maintains order by degrading free energy and producing high entropy waste (also Boltzmann 1886). Free energy may be derived from geologic sources of chemical compounds (chemotrophs), directly from sunlight (phototrophs), or from organic material (heterotrophs).

\subsubsection{Environmental Effects of Biotic Activity}

The resources converted by metabolisms are ultimately derived from the environment. Therefore, each metabolic activity necessarily modifies its environment. For instance, photosynthesis converts carbon dioxide into carbohydrates and oxygen, thereby changing the atmospheric concentrations of carbon dioxide and oxygen. Since carbon dioxide plays an important role as an atmospheric greenhouse gas, the rate of photosynthesis indirectly affects the radiative transfer within the atmosphere. The production of some sulfur-related compounds is related to metabolisms deriving their free energy from sulfate oxidation or reduction (or as a byproduct, as is the case for dimethylsulphide). In the atmosphere, sulfur compounds act as cloud condensation nuclei and impact the rate of formation, the location and brightness of clouds (e.g., Charlson et al. 1987). These examples show the tight linkage between different forms of biotic activity (through their respective metabolisms) and atmospheric functioning at the global scale. Table 1.2 summarizes a few 
in: Kleidon, A. and Lorenz, R. D. (eds.) Non-equilibrium thermodynamics and the production of entropy: life, Earth, and beyond. Springer Verlag, in press.

(C) 2004 Springer Verlag, Heidelberg. ISBN: 3-540-22495-5

1 Entropy Production by Earth System Processes

Table 1.2. Relationships among chemical compounds, biotic activity and atmospheric processes. Also shown are estimates of the global exchange fluxes with the atmosphere related to biotic and abiotic processes under natural conditons

\begin{tabular}{|c|c|c|c|c|}
\hline $\begin{array}{l}\text { Chemical } \\
\text { compound }\end{array}$ & $\begin{array}{l}\text { biotic } \\
\text { activity }\end{array}$ & $\begin{array}{l}\text { atmospheric } \\
\text { process }\end{array}$ & $\begin{array}{l}\text { biotic } \\
\text { exchange } \\
\text { flux } \\
\text { in } 10^{12} \mathrm{~g} / \mathrm{yr}\end{array}$ & $\begin{array}{l}\text { abiotic ex- } \\
\text { change flux } \\
\text { in } 10^{12} \mathrm{~g} / \mathrm{yr}\end{array}$ \\
\hline $\mathrm{CO}_{2}$ & $\begin{array}{l}\text { photosynthesis, } \\
\text { respiration }\end{array}$ & $\begin{array}{l}\text { absorption of terrestrial } \\
\text { radiation }\end{array}$ & $\approx 210,000^{*}$ & $\approx 600^{*}$ \\
\hline $\mathrm{O}_{2}, \mathrm{O}_{3}$ & $\begin{array}{l}\text { photosynthesis, } \\
\text { respiration }\end{array}$ & $\begin{array}{l}\text { absorption of solar radia- } \\
\text { tion in the stratosphere }\end{array}$ & $\approx 32,000^{+}$ & $\approx 0^{+}$ \\
\hline $\mathrm{CH}_{4}$ & methanogenesis & $\begin{array}{l}\text { absorption of } \\
\text { terrestrial radiation }\end{array}$ & $\approx 150$ & $\approx 10^{\pi}$ \\
\hline $\begin{array}{l}\mathrm{NO}, \mathrm{N}_{2} \mathrm{O} \\
\mathrm{NH}_{3}, \mathrm{~N}_{2}\end{array}$ & $\begin{array}{l}\text { nitrogen } \\
\text { fixation, } \\
\text { nitrification, } \\
\text { denitrification }\end{array}$ & $\begin{array}{l}\mathrm{N}_{2} \mathrm{O} \text { : absorption of } \\
\text { terrestrial radiation }\end{array}$ & $\approx 155^{\pi}$ & $<3$ \\
\hline $\begin{array}{l}\mathrm{SO}_{2}, \mathrm{SO}_{4} \\
\mathrm{H}_{2} \mathrm{~S}, \mathrm{DMS}\end{array}$ & $\begin{array}{l}\text { anoxic } \\
\text { photo-synthesis, } \\
\text { sulfate } \\
\text { oxidation and } \\
\text { reduction }\end{array}$ & $\begin{array}{l}\text { cloud formation (acts as } \\
\text { cloud condensation } \\
\text { nuclei) }\end{array}$ & $\approx 20^{\pi}$ & $\approx 162^{\top}$ \\
\hline $\mathrm{H}_{2} \mathrm{O}$ & $\begin{array}{l}\text { transpiration by } \\
\text { terrestrial vege- } \\
\text { tation }\end{array}$ & $\begin{array}{l}\text { water cycling (linked } \\
\text { with greenhouse effect, } \\
\text { cloud formation, heating } \\
\text { by latent heat release) }\end{array}$ & $\approx 3510^{6 \S}$ & $\approx 3810^{6 \S}$ \\
\hline \multicolumn{5}{|c|}{$\begin{array}{l}{ }^{*} \text { Prentice et al. }(2001) \\
+ \text { Jacobson et al. }(2000) \\
\text { ๙ Schlesinger }(1997) \\
\S \text { Kleidon et al. }(2000)\end{array}$} \\
\hline \multicolumn{5}{|c|}{$\begin{array}{l}\text { selected chemical compounds and how they relate to biotic activity and at- } \\
\text { mospheric processes. The last two columns of Table } 1.2 \text { provide estimates of } \\
\text { the global mass exchange fluxes of these compounds for the atmosphere due } \\
\text { to the biota and to abiotic processes. In terms of absolute and relative magni- } \\
\text { tude, the fluxes of carbon dioxide, oxygen and water are those most strongly } \\
\text { modified by biotic activities. These biotic effects lead to a unique composi- } \\
\text { tion of the Earth's atmosphere (Table } 1.3 \text {, also Catling, this volume), with } \\
\text { high concentrations of reactive oxygen reflecting a state far from chemical } \\
\text { equilibrium. }\end{array}$} \\
\hline
\end{tabular}

\subsubsection{The Gaia Hypothesis}

The strong biotic influence on the atmospheric composition leads to a chemical disequilibrium, most notably reflected in the high concentration of reac- 
in: Kleidon, A. and Lorenz, R. D. (eds.) Non-equilibrium thermodynamics and the production of entropy: life, Earth, and beyond. Springer Verlag, in press. (C) 2004 Springer Verlag, Heidelberg. ISBN: 3-540-22495-5

A. Kleidon and R. Lorenz

tive atmospheric oxygen in the Earth's atmosphere (Table 1.3). This chemical disequilibrium is directly linked to the biotic process of photosynthesis that releases oxygen by removing carbon dioxide from the atmosphere. Lovelock (1965) and Hitchcock and Lovelock (1967) suggested to use the existence of such non-equilibrium states of planetary atmospheres as a means to remotely infer the presence of life on other planets.

Based on the notion of Earth's atmospheric composition being far from chemical equilibrium, Lovelock (1972a, b) and Lovelock and Margulis (1974) formulated the controversial Gaia hypothesis, stating "atmospheric homeostasis by and for the biosphere". Associated with the Gaia hypothesis is the notion that biotic feedbacks are primarily negative in nature (which is necessary to maintain homeostasis). Major objections to the Gaia hypothesis include the apparent contradiction to established evolutionary theory, which emphasizes the role of individuals, while the Gaia hypothesis seems to imply a teleological, "goal-seeking" tendency at the planetary scale. Apart from this, it has been pointed out that the hypothesis is ill defined for testing (e.g., Kirchner 1989). The Gaia hypothesis has stimulated a wealth of interdisciplinary research (e.g., Charleston et al. (1987); Schneider and Boston (1991); Schneider et al. (2004)), but is still subject to heated debate (e.g., recent discussion in the journal Climatic Change, Kleidon 2002, 2004; Lenton 2002; Lenton and Wilkinson 2003; Volk 2002, 2003a, b; Kirchner 2002, 2003; Lovelock 2003). While the outcome of this debate is yet inconclusive, it is nevertheless important to note that much of the disagreement can be attributed to a difference in perspective, with the planetary perspective promoted by the Gaia hypothesis sharing many similarities with a viewpoint of non-equilibrium statistical mechanics and maximum entropy production. Two contributions in this volume explicitly connect the MEP principle to the Gaia hypothesis (Kleidon and Fraedrich, this volume; Toniazzo et al., this volume). Kleidon and Fraedrich suggest the existence of a MEP state with respect to absorption of solar radiation due to a minimum in the Earth's planetary albedo, which may result from the biotic influence on atmospheric composition and which would have a Gaia-like outcome. Toniazzo et al. address the question of the relation between an MEP climate system and "Gaian" (i.e., climatically active) biota and of the requirements for self-regulation to operate.

Table 1.3. Atmospheric composition of Earth in comparison to Venus and Mars. Based on data from the National Solar System Data Center (http://nssdc.gsfc.nasa.gov/planetary)

\begin{tabular}{llll}
\hline & Venus & Earth & Mars \\
\hline Surface pressure (bars) & 92 & 1.013 & 0.0064 \\
Oxygen (\%) & $\approx 0$ & 20.95 & 0.13 \\
Carbon dioxide (\%) & 96.5 & 0.0350 & 95.3 \\
Nitrogen (\%) & 3.5 & 78 & 2.7 \\
Water (\%) & 0.002 & $\approx 1$ & 0.0210 \\
\hline
\end{tabular}




\subsubsection{Optimization and Entropy Production Within the Biosphere}

Apart from the extreme case of the Gaia hypothesis, energy-based principles have been suggested for how ecosystems at a smaller scale organize themselves. These include the notion that ecosystems evolve to maximize the energy flux through the system (Lotka 1922a, b; Loreau 1995), or, formulated in slightly different terms, that available energy is degraded at the maximum possible rate, yielding "maximum power" (the maximum power principle of Odum and Pinkerton 1955; Odum 1988). A closely associated observation is that of Ulanowicz and Hannon (1987) who note that vegetated surfaces exhibit cooler surface temperatures and a lower surface albedo, which results in higher rates of entropy production. The trend to increasing entropy production with maturation is interpreted by Ulanowicz and Zickel (this volume) as a trend towards increasing self-organization, which can be quantified by using the concept of ascendency. Schneider and Kay (1994) suggest that ecosystems attain states of maximum dissipation, destroying exergy gradients at a maximum possible rate (with exergy being a measure of the total amount of free energies in the system). This maximization of energy flux should not be seen as only be related to producers (i.e., plants), but in combination with consumption of organic carbon compounds by consumers (e.g., Loreau 1995).

It has also been argued that the emergent optimization of energy fluxes in ecosystems can be understood as the outcome of evolution by natural selection. Alfred Lotka argued in this context that assemblages of organisms can be viewed as "armies of energy tranformers", and the "[evolutionary] advantage must go to those organisms whose energy-capturing devices are most efficient in directing available energy into channels favorable to the preservation of the species" (Lotka 1922a, b).

These principles, in one way or another, are dealing with the rate of entropy production, as discussed in the previous sections. They essentially address the question whether a large number of individual organisms organize themselves in any particular state, which connects these principles to a perspective of statistical mechanics and thermodynamics of non-equilibrium systems.

It is important to point out that when we extend the impact of ecosystems on Earth system functioning to the planetary level, boundary conditions are not fixed. The uptake of carbon dioxide by photosynthesis affects the atmospheric composition, so that we deal with a non-linear system, which, through the inherent diversity within the biosphere, should exhibit many degrees of freedom. This line of reasoning suggests that MEP should potentially be applicable to the interactions of the biosphere with its atmospheric environment, just as atmospheric heat transport interacts with the equator-pole temperature gradient (Catling, this volume; Kleidon and Fraedich, this volume). Another way to look at the applicability of MEP to the biosphere is from a perspective of reproducibility. As discussed in Dewar (this volume), one can consider the reproducibility of a macroscopic system state as the key 
in: Kleidon, A. and Lorenz, R. D. (eds.) Non-equilibrium thermodynamics and the

production of entropy: life, Earth, and beyond. Springer Verlag, in press.

(C) 2004 Springer Verlag, Heidelberg. ISBN: 3-540-22495-5

A. Kleidon and R. Lorenz

idea behind the second law of thermodynamics. As pointed out by Lineweaver (this volume), this translates to the question of which aspects of the Earth's biosphere are reproducible, in terms of the evolution of Earth's surface temperature and life forms (Schwartzman and Lineweaver, this volume) and in terms of biogeochemical evolution of the atmosphere (Catling, this volume).

\subsection{Structure of This Book}

The purpose of this book is to provide a general introduction to the statistical mechanics and thermodynamics of non-equilibrium systems and how it applies to Earth system processes, life on Earth in the context of the evolution of the Universe. It is a synthesis of reviews of previous work in combination with recent developments.

The chapters of this book are organized into three parts. The first part focuses on general and theoretical issues of describing the dynamics of complex systems with special emphasis on entropy production. Eric Chaisson (Chap. 2) provides a general introduction of non-equlibrium thermodynamics in a broader context. His chapter outlines the general direction of the evolution of the Universe to higher rates of energy use and rise in general complexity, from the formation of matter to galaxies, life on Earth, to the evolution of complex human societies. The next chapter introduces the principle of Maximum Entropy Production from a historic perspective. Its author, Garth Paltridge, has been central to the development of the MEP principle and how it relates to atmospheric heat transport. Chap. 4 by Roderick Dewar reviews the information-theory based formulation of statistical mechanics as promoted by Jaynes. As a central piece of Dewar 's chapter, it is shown how the MEP principle can be derived from information theory, how it connects to macroscopic reproducibility, and how the frequently observed phenomenon of self-organized criticality (SOC) of natural and human systems can also be derived from this perspective. Robert Ulanowicz and Michael Zickel discuss the ecological concept of ascendency in Chap. 5 which can be used to quantify the organization of a complex system (such as an ecosystem). This methodology could be used in physical models to quantify the organization of turbulent flow. In Chap. 6, Charles Lineweaver provides a discussion of the universal limitations to the MEP principle, and which aspects of the evolution of the Universe and the Earth's biosphere are macroscopically reproducible (also Schwartzman and Lineweaver, this volume).

The second part of the book focuses on the application of non-equilibrium thermodynamics and the quantification of entropy production in physical systems. Joël Sommeria describes in Chap. 7 a theroretical derivation of MEP from the fluid dynamics of a two-dimensional system. He discusses the emergent behavior of the system and makes connections to the observed red spot of Jupiter as such an emergent feature. Takamitsu Ito and Axel Kleidon (Chap. 8) deal with entropy production by the atmospheric circulation from 
a theoretical viewpoint and how it is simulated by atmospheric General Circulation Models (GCMs). They also provide a demonstration of how the MEP principle can be confirmed with a GCM. In Chap. 9, Olivier Pauluis quantifies the rates of entropy production associated with atmospheric moisture, and discusses the role of dissipation associated with water vapor in the Earth's entropy production budget. Shinya Shimokawa and Hisashi Ozawa (Chap. 10) discuss entropy production associated with the oceanic circulation. They demonstrate the existance of multiple steady states with an oceanic GCM and then show that perturbations of these states generally lead to higher rates of entropy production. Hideaki Miyamoto, Victor Baker, and Ralph Lorenz give an overview of the application of thermodynamics to the formation of river networks and emerging scaling laws in chapter 11. In the last chapter of the second part (Chap. 12), Ralph Lorenz discusses the extension of thermodynamics and MEP to phenomena on phenomena in the solar system and other planets.

The third part of the book deals with the application of thermodynamics to the Earth's biosphere - from a molecular level in Chap. 13 to the Gaia hypothesis (Chap. 17) and the economic activity of the anthroposphere (Chap. 18). In Chap. 13, Davor Juretić and Paško Županović propose the application of thermodynamics to the quantification of rates in initial photosynthetic reactions, pointing out the non-linearities associated with these reactions such that MEP should be applicable. Axel Kleidon and Klaus Fraedrich focus on the large-scale effects of terrestrial vegetation on the physical exchanges of energy and water at the land surface in Chap. 14. They discuss how the MEP principle should be applicable to understand these interactions, and how Gaia-like behavior may result from a MEP state. In Chap. 15, David Catling describes the evolution of the Earth's atmosphere and how it has been affected by the biosphere. He also points out the general energetic advantages of high oxygen concentrations to the biosphere. Chapter 16 by David Schwartzman and Charley Lineweaver covers the biotic evolution on Earth from a viewpoint of temperature constraints and how it is interrelated with global biogeochemical cycles. They argue that the major trends reflected in the early evolution of the Earth's biosphere should be viewed as a deterministic, that is reproducible aspect of biotic evolution on Earth. In Chap. 17, Thomas Toniazzo, Timothy Lenton, Peter Cox and Jonathan Gregory discuss the Gaia hypothesis and how it may relate to the MEP principle. They use the Daisyworld model of Watson and Lovelock (1983) - which was originally developed for demonstrating planetary regulation - to discuss the role of time constants associated with growth for global regulation. The third part of the book closes with chapter 18 by Matthias Ruth. This chapter provides an important linkage to demonstrate the use of thermodynamics to describe the emergent behavior of complex economies. As our Earth system is increasingly dominated by the human species, economic and social processes play an increasing role in shaping the physical environment, and should ultimately affect the rates of entropy production of Earth system processes as well. 
in: Kleidon, A. and Lorenz, R. D. (eds.) Non-equilibrium thermodynamics and the

production of entropy: life, Earth, and beyond. Springer Verlag, in press.

(C) 2004 Springer Verlag, Heidelberg. ISBN: 3-540-22495-5

A. Kleidon and R. Lorenz

As pointed out above, there will doubtless be objections to the MEP principle, notably in determining its applicability domain - when and where is it useful. It is hoped that this volume will encourage that discussion. Ultimately, as MEP becomes a more widely known principle, results will speak for themselves, and help us to provide a general framework to describe the emergent behavior of complex dynamic systems.

\section{References}

Aoki I (1983) Entropy production on the Earth and other planets of solar system. J Phys Soc Jpn 52: 1075-1078.

Bejan A (2000) Shape and Structure, from Engineering to Nature. Cambridge University Press, Cambridge, England. 324pp.

Boltzmann L (1886) Der zweite Hauptsatz der mechanischen Wärmetheorie. Sitzungsber Kaiserl Akad Wiss, Wien, Austria.

Budyko MI (1969) Effects of solar radiation variations on climate of Earth. Tellus 211: 611-619.

Charlson RJ, Lovelock JE, Andreae MO, Warren SG (1987) Oceanic phytoplankton, atmospheric sulphur, cloud albedo, and climate. Nature 326: 655-661.

Dewar RC (2003) Information theory explanation of the fluctuation theorem, maximum entropy production, and self-organized criticality in non-equilibrium stationary states. J Physics A 36: 631-641.

Enquist BJ, Brown JH, West GB (1998) Allometric scaling of plant energetics and population density. Nature 395: 163-165.

Goody R (2000) Sources and sinks of climate entropy. Q J Roy Meteorol Soc 126: $1953-1970$.

Grassl H (1981) The climate at maximum entropy production by meridional atmospheric and oceanic heat fluxes. Q J R Meteorol Soc 107: 153-166.

Hitchcock DR, Lovelock JE (1967) Life detection by atmospheric analysis. Icarus 7: 149-159.

Jacobson MC, Charlson RJ, Rodhe H, Orians GH (2000) Earth system science from biogeochemical cycles to global change. Academic Press, San Diego.

Kirchner JW (1989) The Gaia hypothesis: can it be tested? Rev Geophys 27: 223235.

Kirchner JW (2002) The Gaia hypothesis: Fact, theory, and wishful thinking. Clim Change 52: 391-408.

Kirchner JW (2003) The Gaia hypothesis: conjectures and refutations. Clim Change 58: $21-45$.

Kleidon A (2002) Testing the effect of life on Earth's functioning: How Gaian is the Earth system? Clim Change 52: 383-389.

Kleidon A (2004) Beyond Gaia: Thermodynamics of life and Earth system functioning. Clim Change, in press.

Kleidon A, Fraedrich K, Heimann M (2000) A green planet versus a desert world: estimating the maximum effect of vegetation on land surface climate. Clim Change 44: 471-493. 
in: Kleidon, A. and Lorenz, R. D. (eds.) Non-equilibrium thermodynamics and the production of entropy: life, Earth, and beyond. Springer Verlag, in press.

(C) 2004 Springer Verlag, Heidelberg. ISBN: 3-540-22495-5

1 Entropy Production by Earth System Processes

Kleidon A, Fraedrich K, Kunz T, Lunkeit F (2003), The atmospheric circulation and states of maximum entropy production. Geophys Res Lett 30: 2223, doi:10.1029/2003GL018363.

Lenton TM (2002) Testing Gaia: The effect of life on Earth's habitability and regulation. Clim Change 52: 409-422.

Lenton TM, Wilkinson DM (2003) Developing the Gaia theory. Clim Change 58: $1-12$.

Loreau M (1995) Consumers as maximizers of matter and energy flow in ecosystems. Am Nat 145: 22-42.

Lorenz EN (1960) Generation of available potential energy and the intensity of the general circulation. in: Pfeffer, R.C. (ed), 'Dynamics of Climate', Pergamon Press, Oxford, UK, pp 86-92.

Lorenz RD, Lunine JI, Withers PG, McKay CP (2001) Titan, Mars and Earth: Entropy production by latitudinal heat transport. Geophys Res Lett 28, 415418.

Lotka AJ (1922a) Contribution to the energetics of evolution. Proc Natl Acad Sci USA 8: $147-151$.

Lotka AJ (1922b) Natural selection as a physical principle. Proc Nat Acad Sci USA 8: $151-154$.

Lovelock JE (1965) A physical basis for life detection experiments. Nature 207: $568-570$.

Lovelock JE (1972a) Gaia as seem through the atmosphere. Atmospheric Environment 6: 579-580.

Lovelock JE (1972b) Gaia: A new look at life on Earth. Oxford University Press, Oxford.

Lovelock JE (2003) Gaia and emergence - a response to Kirchner and Volk. Clim Change 57: 1-3.

Lovelock JE, Margulis L (1974) Atmospheric homeostasis by and for the biosphere: the Gaia hypothesis. Tellus 26: 2-10.

North GR, Cahalan RF, Coakley JA (1981) Energy balance climate models. Rev Geophys Space Phys 19: 91-121.

Odum HT (1988) Self-organization, transformity, and information, Science 242: $1132-1139$.

Odum HT, Pinkerton RC (1955) Time's speed regulator: the optimum efficiency for maximum power output in physical and biological systems. Am Sci 43: 331-343.

Ozawa H, Ohmura A, Lorenz RD, Pujol T (2003) The second law of thermodynamics and the global climate system - A review of the maximum entropy production principle. Rev Geophys 41: 1018.

Paltridge GW (1975) Global dynamics and climate - a system of minimum entropy exchange, Q J R Meteorol Soc 101: 475-484.

Paltridge GW (1978) The steady-state format of global climate. Q J Roy Met Soc 104: $927-945$.

Paltridge GW (2001) A physical basis for a maximum of thermodynamic dissipation of the climate system. Q J R Meteorol Soc 127: 305-313.

Peixoto JP, Oort AH, de Almeida M, Tome A (1991) Entropy budget of the atmosphere. J Geophys Res 96: 10, 981-10, 988. 
in: Kleidon, A. and Lorenz, R. D. (eds.) Non-equilibrium thermodynamics and the production of entropy: life, Earth, and beyond. Springer Verlag, in press.

(C) 2004 Springer Verlag, Heidelberg. ISBN: 3-540-22495-5

20 A. Kleidon and R. Lorenz

Prentice IC, Farquhar GD, Fasham MJR, Goulden ML, Heimann M, Jaramillo VJ, Kheshgi HS, Le Quéré C, Scholes RJ, Wallace DWR (2001) The carbon cycle and atmospheric carbon dioxide. in: Climate change 2001: The scientific basis. Contribution of working group I to the third assessment report of the Intergovernmental Panel on Climate Change. Houghton JT, Ding Y, Griggs DJ, Noguer M, van der Linden PG, Dai X, Maskell K, Johnson CA (eds). Cambridge University Press, Cambridge, United Kingdom and New York, NY, USA.

Prigogine I (1962) Introduction to Non-equilibrium Thermodynamics.Wiley Interscience, New York.

Rinaldo A, Maritan A, Colaiori F, Flammini A, Rigon R, Rodriguez-Iturbe I, Banavar JR (1996) Thermodynamics of fractal networks. Phys Rev Lett 76: 33643367.

Rodgers CD (1976) Minimum entropy exchange principle - reply. Q J Roy Meteor Soc 102: 455-457.

Schlesinger WH (1997) Biogeochemistry: an analysis of global change. 2nd edition, Academic Press, San Diego.

Schneider ED, Kay JJ (1994) Life as a manifestation of the second law of thermodynamics. Math Comput Modeling 19: 25-48.

Schneider SH, Boston PJ (1991) Scientists on Gaia. MIT Press, Cambridge, Mass.

Schneider SH, Miller JR, Crist E, Boston PJ (2004) Scientists debate Gaia: The next century. MIT Press, Cambridge, Mass, in press.

Schrödinger E (1944) What is life? The physical aspect of the living cell. Cambridge University Press, Cambridge, UK.

Sellers WD (1969) A global climate model based on the energy balance of the Earth atmosphere system. J Appl Met 8: 392-400.

Shimokawa S, Ozawa H (2001) On the thermodynamics of the oceanic general circulation: Entropy increase rate of an open dissipative system and its surroundings. Tellus 53A: 266-277.

Shimokawa S, Ozawa H (2002) On the thermodynamics of the oceanic general circulation: Irreversible transition to a state with higher rate of entropy production. Q J Roy Meteorol Soc 128: 2115-2128.

Ulanowicz RE, Hannon BM(1987) Life and the production of entropy. Proc R Soc Lond B 232: 181-192.

Volk T (2002) Towards a future for Gaia theory. Clim Change 52: 423-430.

Volk T (2003a) Seeing deeper into Gaia theory - a reply to Lovelock's response. Clim Change 57: 5-7.

Volk T (2003b) Natural selection, Gaia, and inadvertent by-products: A reply to Lenton and Wilkinson's response. Clim Change 58: 13-19.

Watson AJ, Lovelock JE (1983) Biological homeostasis of the global environment: the parable of Daisyworld, Tellus 35B: 284-289.

West GB, Brown JH, Enquist BJ (1999) The fourth dimension of life: fractal geometry and allometric scaling of organisms. Science 284: 167-169. 